\title{
Minimally Invasive Decompression and Physiotherapy for Lumbar Spinal Stenosis in Geriatric Patients
}

\author{
Haydn Hoffman ${ }^{1}$, Shelley S. Bennett ${ }^{2}$, Charles H. Li ${ }^{3}$, Piia Haakana ${ }^{1}$, Daniel C. Lu ${ }^{1}$ \\ 1. Department of Neurosurgery, University of California Los Angeles 2. Department of Physical Therapy, University of \\ California, Los Angeles 3. Department of Neurosurgery, University of California, Los Angeles
}

Corresponding author: Haydn Hoffman, hoffmanh@upstate.edu

\begin{abstract}
Background

Lumbar spinal stenosis (LSS) is the most common indication for spine surgery among the geriatric population. Although decompressive surgery is effective, older patients do not benefit as much as younger patients, and they are frequently excluded from studies assessing postoperative physiotherapy. We sought to evaluate the long-term outcomes after surgery when a novel postoperative physiotherapy regimen was included.

Methods

We performed a retrospective review of patients with LSS greater than 70 years old who underwent lumbar decompressive surgery by the senior author over the past five years. We evaluated patients who participated in a novel postoperative physiotherapy regimen involving four phases of rehabilitation aimed at progressively independent ambulation. The visual analog scale (VAS), lower extremity motor strength, and functional independence measure (FIM) were collected preoperatively and after physiotherapy to measure outcomes.
\end{abstract}

Results

Ten consecutive patients with an average age of 83 years (range: 71 - 96) met the inclusion criteria. Nine patients underwent minimally invasive laminotomies at L4-L5 and one underwent a laminotomy at L3-L4. The average follow-up time was 41.9 months. The preoperative mean VAS was 7.35, and at the end of the study, it was $1.7(\mathrm{p}=0.005)$. Three of the four patients with preoperative motor deficits improved. The median transfer and locomotion subscores of the FIM were six preoperatively and increased to seven postoperatively. Neither of these improvements was significant.

Received 04/16/2018

Review began 04/16/2018

Review ended 06/08/2018

Published 06/11/2018

๑ Copyright 2018

Hoffman et al. This is an open access article distributed under the terms of the Creative Commons Attribution License CC-BY 3.0., which permits unrestricted use, distribution, and reproduction in any medium, provided the original author and source are credited.

\section{Conclusions}

Patients older than 70 years undergoing decompressive surgery and a novel postoperative physiotherapy regimen experienced significant reductions in pain. Independence also increased; however, this did not reach statistical significance.

Categories: Physical Medicine \& Rehabilitation, Neurosurgery

Keywords: spinal stenosis, lumbar decompression, postoperative rehabilitation

\section{Introduction}

Degenerative lumbar spinal stenosis (LSS) is one of the most common etiologies of back pain and radiculopathy affecting the geriatric population. Given the aging population of the United States, there has been a $23 \%$ increase in surgical operations performed for LSS [1]. In recent years, there has been an increased focus on surgical interventions for LSS in older patients spurred by advances in minimally invasive techniques that minimize the morbidity of back surgery [2-4]. There is limited outcome data on postoperative physiotherapy for LSS in the geriatric population, however [5]. Recent data suggest that postoperative rehabilitation following decompressive surgery is effective at reducing pain and improving quality of life and functional status, but geriatric patients have been largely excluded from these studies [67]. Furthermore, specific rehabilitation regimens for the geriatric population following surgery for LSS have not been described, and the nature of those for the general population is heterogeneous [8].

Given the lack of data regarding postoperative rehabilitation after lumbar decompressive surgery in geriatric patients, we sought to evaluate the efficacy and feasibility of a novel physiotherapy regimen for this population. The proposed regimen included four phases aimed at restoring ambulation: (1) extremity and 


\section{Cureus}

core muscle conditioning, (2) balance and stance training, (3) cardiovascular conditioning, and (4) sit-tostand training. We were interested in the pain and functional status of these patients following completion of rehabilitation. This data will guide surgeons and physical therapists in the postoperative management of older patients with LSS.

\section{Materials And Methods}

\section{Patient recruitment}

After obtaining institutional review board approval, patients with LSS presenting to the clinic of the senior author were considered for inclusion in the study. Diagnosis of LSS was confirmed by findings on magnetic resonance imaging and clinical correlation of the patient's symptoms. Inclusion criteria included age $>70$ years, symptoms of radiculopathy and/or neurogenic claudication, radiographic evidence of spinal stenosis, and medical clearance for surgery. Exclusion criteria included a history of prior lumbar spine surgery, spondylolisthesis, scoliosis, and orthopedic or peripheral neurologic comorbidities that could have confounded ambulation status. All patients underwent minimally invasive laminotomy by the senior author according to a technique previously described (Video 1) [9]. This surgical approach was performed through a small $1.5 \mathrm{~cm}$ incision. In most cases, patients were able to go home on the day of their procedure.

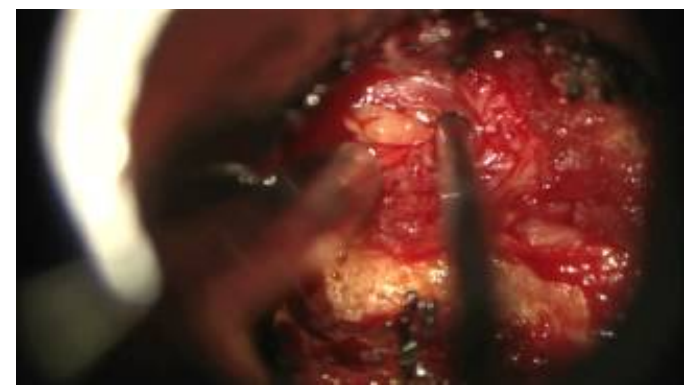

VIDEO 1: Minimally invasive laminotomy

[9]

View video here: http://youtu.be/aXyZ2FJMh2s

\section{Physiotherapy regimen}

The physiotherapy was designed and performed individually and face-to-face by a single physical therapist in order to eliminate potential inter-rater disagreement. The physiotherapy consisted of a weekly 90 minute adaptive, graded regimen that was divided into four phases to address specific deficits with the final goal of restoring independent standing and ambulatory abilities (Table 1). Adherence to therapy sessions was assessed by the physical therapist and all participants adhered to this schedule.

\begin{tabular}{|c|c|c|}
\hline Phase & Stage & Recommended postoperative start date \\
\hline 1 & Extremity and core muscle conditioning & 1 week \\
\hline 2 & Balance and stance training & 1 month \\
\hline 3 & Cardiovascular conditioning and gait training & 2 months \\
\hline 4 & Sit-to-stand training & 3 months \\
\hline
\end{tabular}

TABLE 1: Phases of Rehabilitation

The timing of each phase was dependent on accomplishing the tasks in the preceding phase. Phase 1 consisted of extremity and core muscle strengthening to build the necessary substrates for motor function. This began one week postoperatively and was continued for the duration of the regimen. Initially, exercises were performed in the supine position and then were advanced to the standing stationary position. Adjunctive functional electrical stimulation (FES) was carried out as described in Table 2. 


\section{Cureus}

\begin{tabular}{|c|c|c|}
\hline Exercise & Performance & Goal \\
\hline Supine exercises & & \# of repetitions \\
\hline $\begin{array}{l}\text { Bilateral ankle } \\
\text { pumps }\end{array}$ & With legs elevated in bed, gently flex and extend ankles & 10 \\
\hline $\begin{array}{l}\text { Hip } \\
\text { abduction/adduction }\end{array}$ & $\begin{array}{l}\text { With patient lying on side, asked to abduct and adduct hips. Switch } \\
\text { sides. }\end{array}$ & 10 \\
\hline Bilateral heel slides & $\begin{array}{l}\text { With legs fully extended, heels are moved up towards the buttocks } \\
\text { as far as comfortable }\end{array}$ & 10 \\
\hline $\begin{array}{l}\text { Sitting or standing } \\
\text { exercises }\end{array}$ & & No. repetitions \\
\hline $\begin{array}{l}\text { Sit to stand at } \\
\text { walker }\end{array}$ & $\begin{array}{l}\text { With maximum assistance, the patient is asked to stand with } \\
\text { support of walker }\end{array}$ & $\begin{array}{l}\text { As many as possible; track repetitions } \\
\text { over time }\end{array}$ \\
\hline Bilateral heel raises & $\begin{array}{l}\text { With legs flexed, the patient is asked to raise heels while seated in } \\
\text { walker }\end{array}$ & 10 \\
\hline $\begin{array}{l}\text { Bilateral knee } \\
\text { extension/flexion }\end{array}$ & Patient is asked to flex and extend knees while seated in walker & 10 \\
\hline Restorator & $\begin{array}{l}\text { Upper extremity strengthening machine, the patient is asked to } \\
\text { rotate device with arms }\end{array}$ & $\begin{array}{l}\text { As many as possible in } 3 \text { minutes at a } \\
\text { speed of } 4.0 \text { with medium resistance }\end{array}$ \\
\hline Ball squeezes & $\begin{array}{l}\text { Rubber ball placed between patient's legs; patient asked to flex and } \\
\text { relax against ball resistance }\end{array}$ & 10 for 3 sets \\
\hline $\begin{array}{l}\text { Isometric } \\
\text { quadriceps } \\
\text { squeezes }\end{array}$ & $\begin{array}{l}\text { Patient started without resistance and eventually progressed to } 2.5 \\
\text { lbs ankle weights for isometric quad squeezes }\end{array}$ & 5 for 10 seconds each contraction \\
\hline $\begin{array}{l}\text { Scapular } \\
\text { contractions }\end{array}$ & Isometric contraction and relaxation of scapular muscles & 10 \\
\hline Bilateral marching & $\begin{array}{l}\text { With assistance, the patient is asked to march in place to work on } \\
\text { hip flexion; patient is progressed to marching with ankle weights }\end{array}$ & 10 \\
\hline $\begin{array}{l}\text { Functional electrical } \\
\text { stimulation }\end{array}$ & & Time (minutes) \\
\hline Gastrocnemius & Stimulation for 40 minutes at $20-30 \mathrm{~Hz}$ intensity; vitals monitored & 40 \\
\hline Gluteus maximus & Stimulation for 40 minutes at $20-30 \mathrm{~Hz}$ intensity; vitals monitored & 40 \\
\hline Tibialis anterior & Stimulation for 40 minutes at $20-30 \mathrm{~Hz}$ intensity; vitals monitored & 40 \\
\hline Hamstrings & Stimulation for 40 minutes at $20-30 \mathrm{~Hz}$ intensity; vitals monitored & 40 \\
\hline
\end{tabular}

\section{TABLE 2: Phase 1 Exercises and Functional Electric Stimulation Protocol}

Phase 2 was comprised of aquatic balance and stance training to accommodate the upright position. Since phase 2 began approximately one month postoperatively, surgical incisions had healed sufficiently to allow for safe exposure to water. Aquatic therapy was favored over ground exercises because it reduced axial load on the spine and had been suggested to be better tolerated in the geriatric population [10]. Phase 2 therapy sessions lasted approximately 40 minutes a day and were focused on standing and locomotion with the goal of increasing the duration of standing with minimal assistance.

Phase 3 involved cardiovascular conditioning and gait training to accommodate the cardiovascular demand of ambulation. This began with the prerequisite that the patient could stand with or without assistance for at least 10 seconds. Patients were instructed to walk in a straight line (with the assistance of a front-wheel walker as needed) with the goal of increasing the maximum distance tolerated before needing to rest.

Phase 4 involved sit-to-stance training. 


\section{Cureus}

\section{Outcome measurement and data analysis}

Outcome data were obtained by the physical therapist. The visual analog scale (VAS) was used to assess the severity of each patient's pain before surgery and at the end of phase 4 . When a patient had both back and leg pain, the source of pain most concerning to the patient was considered. In addition, each patient's strength was measured before surgery and at the end of phase 4 by testing the muscles associated with each lumbar and sacral nerve root. Strength was measured on a scale from 0 to 5 as defined by the Medical Research Council Scale for Muscle Strength. The functional independence measure (FIM) is a reliable tool for evaluating a subject's disability in the domains of self-care, sphincter management, mobility, and locomotion. It was used to evaluate each patient before surgery and at the end of phase 4 . Wilcoxon signedrank test was used to compare scores before and after surgery.

Electromyography (EMG) was performed on patient \#10 at 10 and 16-month postoperative time intervals to measure his improvement over the course of sit-to-stand training. Data was collected at 2,000 Hz and subsequently processed in Matlab (Mathworks, Natick, MA). The following were sequentially applied to the data: direct current (DC) offset removal, 5th-order Butterworth bandpass filter (10 - $500 \mathrm{~Hz})$, rectification, and linear envelope. The EMG signals were analyzed to compute the amplitude, timing, and duration of individual electrical bursts. Root-mean-square (RMS) EMG activity of the rectus femoris (RF), hamstrings (HAM), tibialis anterior (TA), and medial gastrocnemius (MG) muscles were calculated. EMG amplitudes were not normalized because the same patient's data was compared six months apart and muscles were analyzed individually.

\section{Results}

A total of 10 patients (three females, seven males) met the inclusion criteria and were included in the study. The average age was 83 years (range: 71 - 96). Additional demographic data is shown in Table 3. Six patients underwent single-level minimally invasive laminotomies at L4-L5 and one underwent a single level

laminotomy at L3-L4. Three patients underwent surgery at multiple levels, as shown in Table 3. There were no intraoperative complications and no patients required reoperation. The average duration of surgery was 62 minutes (range: 38 - 120). The average follow-up time was 41.9 months (range: 24 - 60). No adverse effects from the physiotherapy were observed in the cohort.

\begin{tabular}{|c|c|c|c|c|}
\hline Patient no. & Age & Gender & Procedure levels & Follow-up time (months) \\
\hline 1 & 71 & $F$ & L4-5 & 60 \\
\hline 2 & 82 & M & L3-4 & 36 \\
\hline 3 & 78 & M & L2-3, L4-5 & 38 \\
\hline 4 & 77 & $\mathrm{~F}$ & L4-5 & 34 \\
\hline 5 & 92 & M & L4-5 & 26 \\
\hline 6 & 84 & $\mathrm{~F}$ & L4-5 & 57 \\
\hline 7 & 89 & M & L4-5 & 54 \\
\hline 8 & 85 & M & L4-5 & 50 \\
\hline 9 & 76 & M & L4-5, L5-S1 & 40 \\
\hline 10 & 96 & $M$ & L3-4, L4-5 & 24 \\
\hline
\end{tabular}

\section{TABLE 3: Patient Demographics}

F: female; M: male; L: lumbar

The mean VAS score for the group was 7.35 (standard deviation $(\mathrm{SD})=1.29$ ) before surgery and $1.7(\mathrm{SD}=1.7)$ at the end of the study (Figure 1$)$. The decrease was statistically significant $(\mathrm{p}=0.005)$. The mean reduction in the VAS score at the end of the study was $5.65(\mathrm{SD}=1.9)$. Each patient experienced improvement in their VAS score after surgery. 


\section{Cureus}

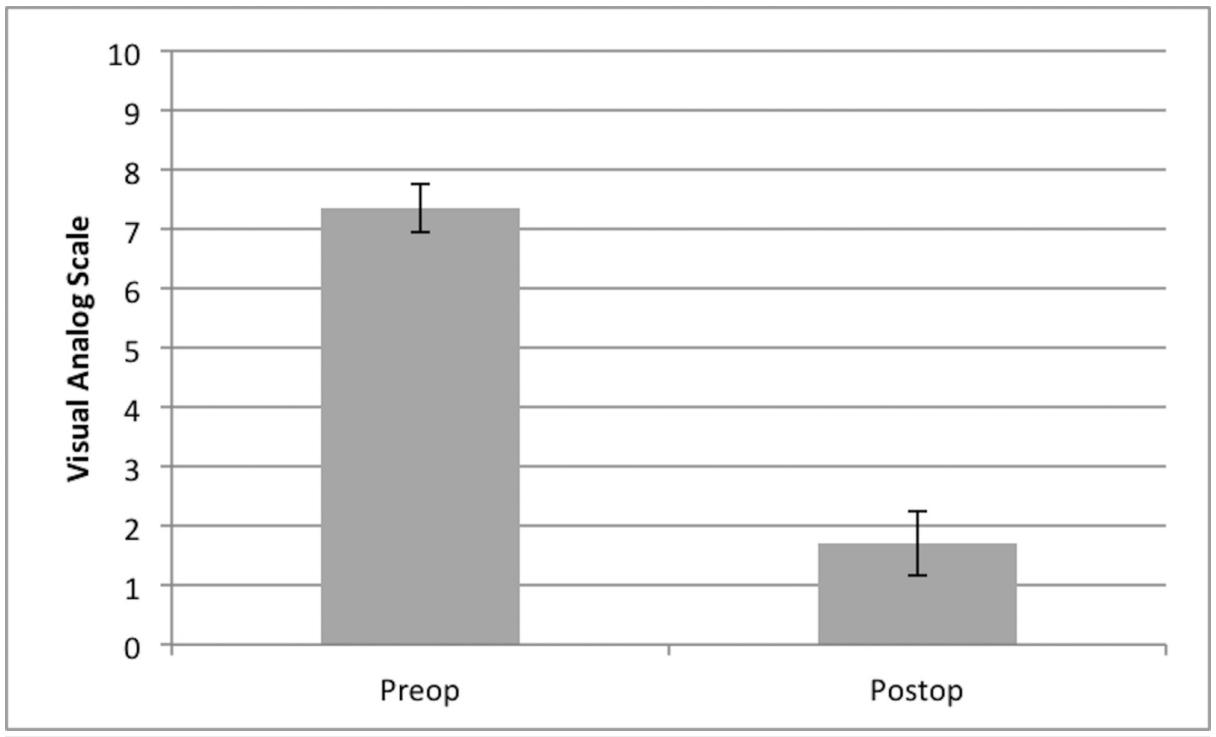

FIGURE 1: Mean visual analog scale scores decreased significantly after surgery and physiotherapy $(p=0.005)$

Error bars indicate standard error of the mean.

Preoperatively, four patients had objective motor weakness in the lower extremities. Subject \#4 had 4/5 strength with right-sided dorsiflexion, which returned to full strength at the end of the study. Subject \#7 had $4 / 5$ strength with right-sided knee extension, and she also returned to full strength at the end of the study. Preoperatively, Subject \#9 had 3/5 strength with left-sided extensor hallucis longus, and this was unchanged at the end of the study. Finally, Subject \#10 had 1/5 strength preoperatively with right-sided dorsiflexion, and this improved to $4 / 5$ at the end of the study. His left-sided dorsiflexion was $3 / 5$ preoperatively, and this improved to full strength. None of the patients experienced a decrease in strength after surgery.

FIM scores were available for nine of the 10 patients. The patient with missing FIM data was excluded from this analysis. Preoperative deficits were only in the domains of transfers and locomotion and were compared to postoperative scores in Figure 2. The median preoperative transfer score was 6 (range: 5 - 7) and increased to 7 (range: $6-7$ ) at the end of the study. The median preoperative locomotion score was 6 (range: $3-7$ ) and increased to 7 (range: 6 - 7). Neither of these improvements was statistically significant. Both patients who required assistance ambulating preoperatively (scores of 3 and 4 ) no longer required assistance at the end of the study (both increased to scores of 6).

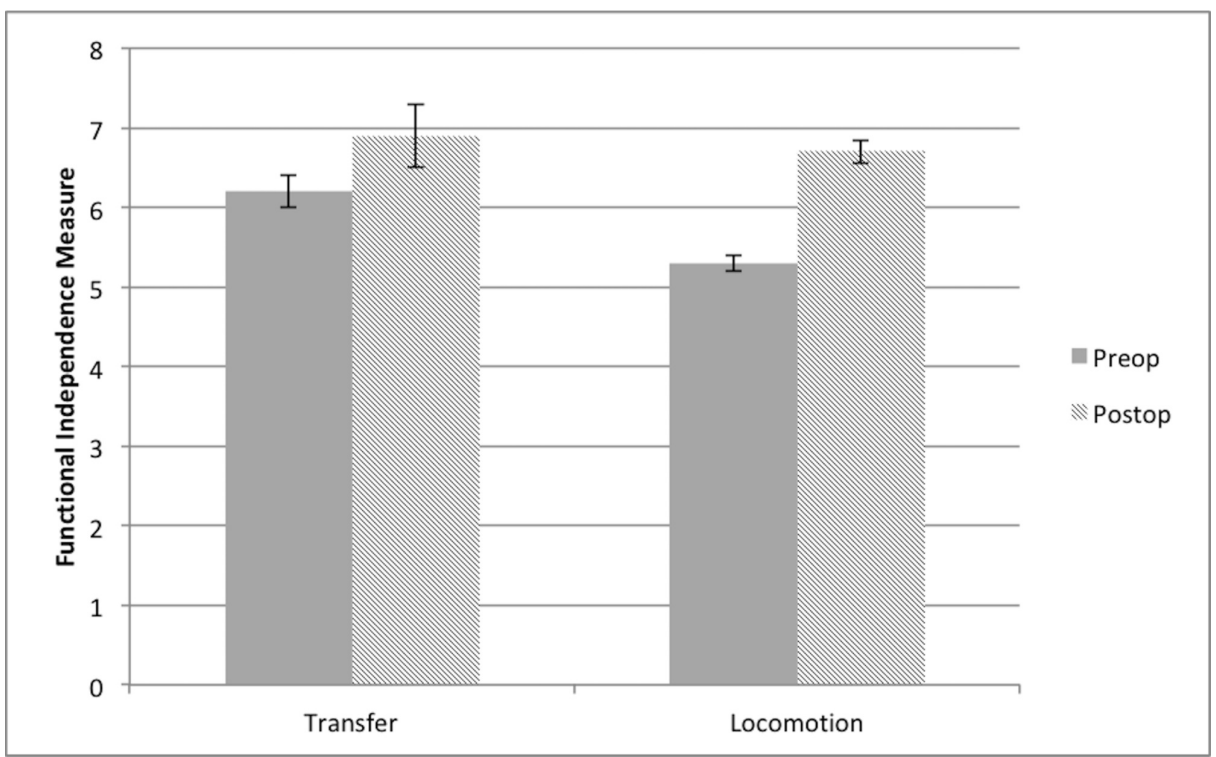

FIGURE 2: Functional independence measure scores

Mean functional independence measure scores for transfer and locomotion domains increased after surgery 
EMG recordings were available for patient \#10 at 10 and 16 months postoperatively. At the latter time point, there were increases in the RMS amplitudes throughout the right and left muscle groups (except the left TA) when the patient stood up (Figure $3 A$ ). The improvement was more pronounced on the patient's right side, corresponding to his deficit prior to surgery. Additionally, the frequency of EMG activity was measured during the patient's sit-to-stand training and a similar increase in amplitudes was observed at the 16-month time point (Figure 3B-C). This was most evident in the right MG.

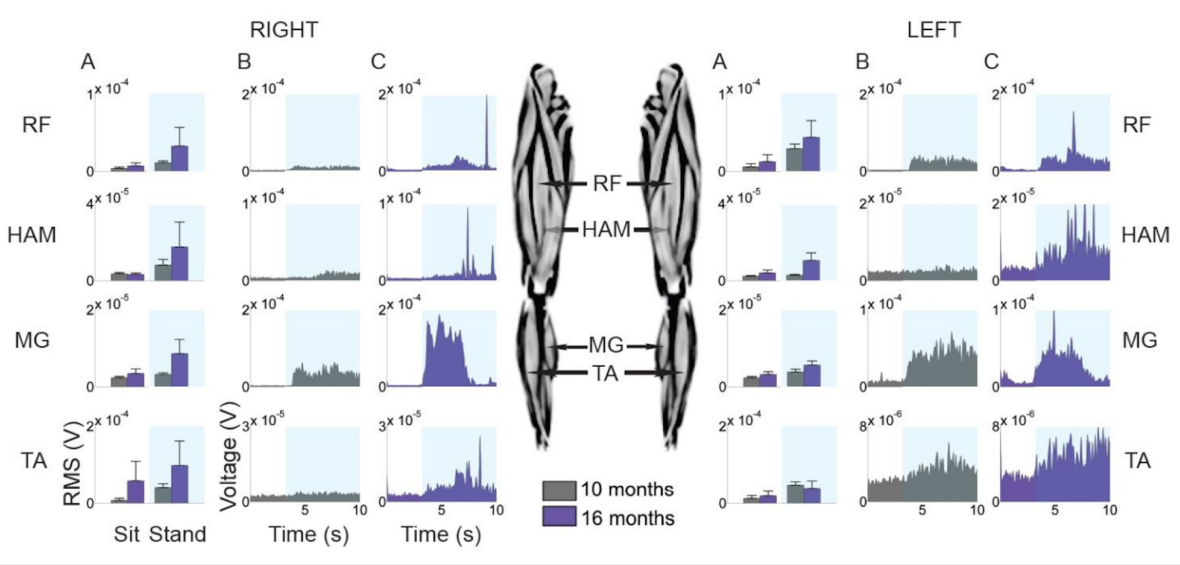

FIGURE 3: EMG recordings during sit-to-stand training for patient \#10 at 10 months and 16 months postoperatively. Root-mean-square value with position (a) and absolute amplitude over the duration of the activity at 10 months (b) and 16 months (c) are provided.

EMG: electromyography; RF: rectus femoris; HAM: hamstrings; TA: tibialis anterior; MG: medial gastrocnemius; RMS: root-mean-square.

\section{Discussion}

LSS is the most common indication for spinal surgery in patients older than 65 years [3]. Surgery should not be withheld from this population based on age alone because it has been shown to be superior to nonoperative therapy for improving pain, disability, and quality of life [11]. Given that almost 25\% of patients undergoing decompression for LSS do not benefit and require reoperation [12], maximizing the benefits of surgery is important. This is even more crucial in the older population, which is more vulnerable to postoperative complications, comorbidity, and increasingly advanced pathology. Rinh et al. found that octogenarians did not improve as much as younger patients with regard to pain and self-rated progress after surgery for LSS [11]. In addition, the postoperative complication rate for older patients undergoing lumbar spine surgery has been reported to be higher than those less than 65 years old [13-16]. In those older than 75 years, the wound complication rate has been reported to be as high as $28 \%$, and the systemic complication rate as high as $18 \%$ [17]. When obesity is present, the reoperation rate is as high as $9.6 \%$ [18]. Thus, the geriatric population merits special attention to improve outcomes.

In the general population, postoperative physiotherapy for LSS has frequently been shown to be helpful in the recovery of strength and movement, as well as the resolution of pain [7, 19]. Depending on the primary outcome measure and follow-up time, however, the benefit is inconsistent. Mannion et al. performed a randomized controlled trial of 159 patients who underwent decompressive surgery for LSS with or without supervised postoperative physiotherapy [20]. The changes in pain and disability levels up to 24 months after surgery were not different between the groups. Given the discrepancy in improvement after decompression for LSS in geriatric patients and the lack of evidence regarding postoperative physiotherapy in this population, we sought to evaluate the outcomes of a physiotherapy regimen that was initiated after surgery for LSS.

Significant improvement in pain was noted in our study group. The mean VAS score decreased by a total of 5.65 points after surgery and physiotherapy. This compares favorably to large cohorts of geriatric patients receiving decompression without physiotherapy. Rosen et al. showed a 3.5 and 3.4 point decrease in back 
and leg pain, respectively, in 50 patients older than 75 years [21]. In a retrospective series of 125 elderly patients followed five years postoperatively, the average reduction in the VAS score was 5.1 points [22]. Without a randomized trial, it is not possible to determine whether the improvements seen in our series were augmented by physiotherapy or simply the effect of surgery. Regardless, it is encouraging that our series experienced such a high level of improvement.

We did not observe significant differences in FIM scores after surgery and physiotherapy. This was likely due to the small sample size and the fact that preoperatively most patients only had mild reductions in their independence. Both patients who required assistance ambulating preoperatively demonstrated independence at the end of phase 4, however. All patients showed improvement in at least one domain at the end of the study. The domains of self-care and sphincter control were omitted because none of the patients endorsed limitations in performing these activities. Similarly, preoperative deficits in strength were minor and heterogeneous, precluding statistical analysis. Only one patient who had a deficit did not improve at the end of the study, likely because this was longstanding over several years.

Limited data exist to guide the development of a physiotherapy regimen for geriatric patients with LSS, and the data that does exist is largely derived from non-surgical populations. Furthermore, no standard of care exists for the delivery of physiotherapy for LSS [23], and when regimens are evaluated, the actual amount of therapy delivered is frequently not standardized or adequately described [24-25]. Likewise, the modalities applied in the community are heterogeneous [26]. The phases of rehabilitation in our regimen were designed to represent an incremental progression of ambulatory ability because ambulation is a primary concern of older patients with neurogenic claudication [23]. Strengthening and aerobic conditioning are central components of each phase, which have been shown to be effective for reducing pain and disability in patients with LSS [27]. The phases were designed such that it would have been difficult to achieve competence in a higher phase without having accomplished the preceding phases. For example, it is difficult to achieve stepping and gait (phase 3) without having muscle strength (phase 1) and balance (phase 2). Dividing ambulation into distinct goals can motivate subjects' participation. Compliance is likely to be more readily obtained if small milestones (i.e., phases) are successfully obtained over time [28], and we observed this in our study group. This is especially pertinent to the geriatric population, in which deconditioning and comorbidity may limit participation. A randomized trial is needed to assess the efficacy of this approach compared to other modes of physiotherapy.

This study is limited by the small sample receiving our intervention, which was not sufficient to detect small improvements in functional independence. Regardless, we were able to demonstrate a statistically significant improvement in pain that compared favorably to other published data [21-22]. Additionally, we did not include a control group (i.e., surgery without postoperative rehabilitation) to elucidate what amount of improvement, if any, was attributable to the physiotherapy. The generalizability of this study is limited by the fact that interventions were carried out by a single neurosurgeon and physical therapist at a single institution. This study does, however, suggest that postoperative rehabilitation is feasible in an older population with LSS and provides a regimen that can be tolerated with good compliance. A randomized trial is warranted to determine its efficacy.

\section{Conclusions}

In a series of patients older than 70 years with LSS, significant improvements in pain were observed with minimally invasive decompressive surgery and a graded postoperative physiotherapy regimen focused on improving ambulation.

\section{Additional Information \\ Disclosures}

Human subjects: Consent was obtained by all participants in this study. University of California Los Angeles Institutional Review Board issued approval 10-001174. Animal subjects: All authors have confirmed that this study did not involve animal subjects or tissue. Conflicts of interest: In compliance with the ICMJE uniform disclosure form, all authors declare the following: Payment/services info: All authors have declared that no financial support was received from any organization for the submitted work. Financial relationships: All authors have declared that they have no financial relationships at present or within the previous three years with any organizations that might have an interest in the submitted work. Other relationships: All authors have declared that there are no other relationships or activities that could appear to have influenced the submitted work.

\section{References}

1. Parker SL, Fulchiero EC, Davis BJ, et al.: Cost-effectiveness of multilevel hemilaminectomy for lumbar stenosis-associated radiculopathy. Spine J. 2011, 11:705-11. 10.1016/j.spinee.2011.04.024

2. Aleem IS, Rampersaud YR: Elderly patients have similar outcomes compared to younger patients after minimally invasive surgery for spinal stenosis. Clin Orthop Relat Res. 2014, 472:1824-30. 10.1007/s11999013-3411-y

3. Deyo RA, Mirza SK, Martin BI, et al.: Trends, major medical complications, and charges associated with 
surgery for lumbar spinal stenosis in older adults. JAMA. 2010, 303:1259-65. 10.1001/jama.2010.338

4. McGirt MJ, Parker SL, Hilibrand A, et al.: Lumbar surgery in the elderly provides significant health benefit in the US health care system: patient-reported outcomes in 4370 patients from the N2QOD registry. Neurosurgery. 2015, 77:S125-35. 10.1227/NEU.0000000000000952

5. Delitto A, Piva SR, Moore CG, et al.: Surgery versus nonsurgical treatment of lumbar spinal stenosis: a randomized trial. Ann Intern Med. 2015, 162:465-73. 10.7326/M14-1420

6. Chen CY, Chang CW, Lee ST, et al.: Is rehabilitation intervention during hospitalization enough for functional improvements in patients undergoing lumbar decompression surgery? A prospective randomized controlled study. Clin Neurol Neurosurg. 2015, 129:S41-46. 10.1016/S0303-8467(15)30011-1

7. McGregor AH, Probyn K, Cro S, et al.: Rehabilitation following surgery for lumbar spinal stenosis: a Cochrane review. Spine (Phila Pa 1976). 2014, 39:1044-54. 10.1097/BRS.0000000000000355

8. Oosterhuis T, Costa LO, Maher CG, et al.: Rehabilitation after lumbar disc surgery. Cochrane Database Syst Rev. 2014, March:CD003007. 10.1002/14651858.CD003007.pub3

9. Kimball J, Yew A, Lu DC: Minimally invasive surgery for lumbar microdiscectomy. Neurosurg Focus. 2013, 35:Video 15. 10.3171/2013.V2.FOCUS13219

10. Baena-Beato PÁ, Artero EG, Arroyo-Morales M, et al.: Aquatic therapy improves pain, disability, quality of life, body composition and fitness in sedentary adults with chronic low back pain. A controlled clinical trial. Clin Rehabil. 2014, 28:350-60. 10.1177/0269215513504943

11. Rihn JA, Hilibrand AS, Zhao W, et al.: Effectiveness of surgery for lumbar stenosis and degenerative spondylolisthesis in the octogenarian population: analysis of the Spine Patient Outcomes Research Trial (SPORT) data. J Bone Joint Surg Am. 2015, 97:177-85. 10.2106/JBJS.N.00313

12. Mannion AF, Denzler R, Dvorak J, Grob D: Five-year outcome of surgical decompression of the lumbar spine without fusion. Eur Spine J. 2010, 19:1883-91. 10.1007/s00586-010-1535-2

13. Shabat S, Arinzon Z, Folman Y, et al.: Long-term outcome of decompressive surgery for lumbar spinal stenosis in octogenarians. Eur Spine J. 2008, 17:193-98. 10.1007/s00586-007-0514-8

14. Carreon LY, Puno RM, Dimar JR 2nd, et al.: Perioperative complications of posterior lumbar decompression and arthrodesis in older adults. J Bone Joint Surg Am. 2003, 85-A:2089-92.

15. Raffo CS, Lauerman WC: Predicting morbidity and mortality of lumbar spine arthrodesis in patients in their ninth decade. Spine (Phila Pa 1976). 2006, 31:99-103. 10.1097/01.brs.0000192678.25586.e5

16. Sobottke R, Aghayev E, Röder C, et al.: Predictors of surgical, general and follow-up complications in lumbar spinal stenosis relative to patient age as emerged from the Spine Tango Registry. Eur Spine J. 2012, 21:411-17. 10.1007/s00586-011-2016-y

17. Wang MY, Green BA, Shah S, et al.: Complications associated with lumbar stenosis surgery in patients older than 75 years of age. Neurosurg Focus. 2003, 14:e7. 10.3171/foc.2003.14.2.8

18. Gepstein R, Shabat S, Arinzon ZH, et al.: Does obesity affect the results of lumbar decompressive spinal surgery in the elderly?. Clin Orthop Relat Res. 2004, 426:138-44. 10.1097/01.blo.0000141901.23322.98

19. Chen CY, Chang CW, Lee ST, et al.: Is rehabilitation intervention during hospitalization enough for functional improvements in patients undergoing lumbar decompression surgery? A prospective randomized controlled study. Clin Neurol Neurosurg. 2015, 129:S41-46. 10.1016/S0303-8467(15)30011-1

20. Mannion AF, Denzler R, Dvorak J, et al.: A randomised controlled trial of post-operative rehabilitation after surgical decompression of the lumbar spine. Eur Spine J. 2007, 16:1101-17. 10.1007/s00586-007-0399-6

21. Rosen DS, O'Toole JE, Eichholz KM, et al.: Minimally invasive lumbar spinal decompression in the elderly: outcomes of 50 patients aged 75 years and older. Neurosurgery. 2007, 60:503-509. 10.1227/01.NEU.0000255332.87909.58

22. Bouras T, Stranjalis G, Loufardaki M, et al.: Predictors of long-term outcome in an elderly group after laminectomy for lumbar stenosis. J Neurosurg Spine. 2010, 13:329-34. 10.3171/2010.3.SPINE09487

23. Lyle S, Williamson E, Darton F, et al.: A qualitative study of older people's experience of living with neurogenic claudication to inform the development of a physiotherapy intervention. Disabil Rehabil. 2017, 39:1009-17. 10.1080/09638288.2016.1177611

24. Macedo LG, Hum A, Kuleba L, et al.: Physical therapy interventions for degenerative lumbar spinal stenosis: a systematic review. Phys Ther. 2013, 93:1646-60. 10.2522/ptj.20120379

25. Reiman MP, Harris JY, Cleland JA: Manual therapy interventions for patients with lumbar spinal stenosis: a systematic review. NZ J Physiother. 2009, 37:17-28.

26. Tomkins CC, Dimoff KH, Forman HS, et al.: Physical therapy treatment options for lumbar spinal stenosis . J Back Musculoskelet Rehabil. 2010, 23:31-37. 10.3233/BMR-2010-0245

27. Goren A, Yildiz N, Topuz O, et al.: Efficacy of exercise and ultrasound in patients with lumbar spinal stenosis: a prospective randomized controlled trial. Clin Rehabil. 2010, 24:623-31. 10.1177/0269215510367539

28. van der Wardt V, Hancox J, Gondek D, et al.: Adherence support strategies for exercise interventions in people with mild cognitive impairment and dementia: a systematic review. Prev Med Rep. 2017, 7:38-45. 10.1016/j.pmedr.2017.05.007 